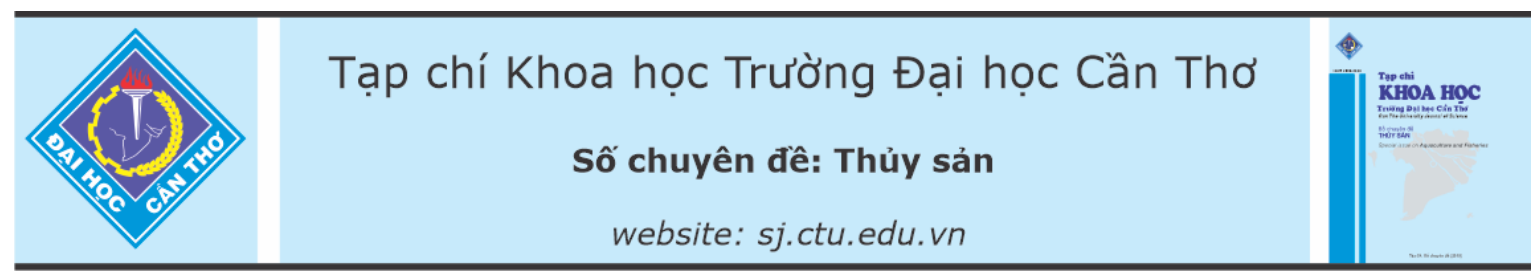

DOI:10.22144/ctu.jvn.2021.079

\title{
TRIỂN KHAI GIẢI THUẠTT DÒ ĐIỂM CÔNG SUÂT CỬC ĐẠI CHO HỆ THỐNG DÙNG NĂNG LƯợNG MặT TRỜI TRONG NUÔI TRỔNG THỦY SẢN
}

\author{
Hồ Phạm Thành Tâm ${ }^{1}$, Nguyễn Thái $\mathrm{Nghe}^{2}$, Nguyễn Chí Ngôn ${ }^{1}$ và Trần Thanh Hùng ${ }^{1 *}$ \\ ${ }^{1}$ Khoa Công nghệ, Truờng Đại học Cần Tho' \\ ${ }^{2}$ Khoa Công nghệ Thông tin và Truyền thông, Truờng Đại học Cần Tho \\ *Nguời chịu trách nhiệm về bài viết: Trần Thanh Hùng (email: tthung@ctu.edu.vn)
}

\section{Thông tin chung:}

Ngày nhận bài: $22 / 02 / 2021$

Ngày nhận bài sủa: 24/03/2021

Ngày duyệt đăng: 01/06/2021

\section{Title:}

Implementation of maximum power point tracking algorithm for solar energy systems in aquaculture

\section{Tù khóa:}

Điểm công suất cực đại, năng luợng mặt tròii, quạt nước ao tôm

\section{Keywords:}

Maximum power point, paddle wheel aerator, solar power

\begin{abstract}
Using solar energy in aquaculture is one of the encouraged solutions in sustainable development. This paper aims to introduce and provide a method of implementing the algorithm to detect and track the maximum power point of photovoltaic panels (solar panels) based on the conductivity of the panel (Incremental Conductance Maximum Power Point Tracking - Ind. Cond. MPPT). This is an important part of creating a basis for deploying solar energy in aquaculture ponds, especially addressing the energy needs of the paddle wheel aerator for shrimp ponds (for oxygen supply and water stirring), because it helps maximize the extracted energy from solar panels. Based on the theory of the electrical model of the photovoltaic panel, the Incremental Conductance algorithm is implemented in the interleaved boost DC-DC converter topology controlled by a programmable digital controller (STM32F1 microcontroller unit). The results of the implementation show that the voltage at the maximum power point of the panel array is always stably maintained, the extracted power is controlled to track the maximum available power of the panel array, the system with the Ind. Cond. The MPPT algorithm is stable and does not get stuck into the transfer characteristic transient region of the DC-DC converter.
\end{abstract}

\section{TÓM TẮT}

Sử dụng năng luợng mặt trời trong nuôi trồng thủy sản là giải pháp phát triển bền vững mà nhà nước đang khuyến khích. Bài báo này nhằm muc tiêu giới thiệu và cung cấp phuơng pháp triển khai giải thuật dò tìm và bám theo điểm công suất cực đại của tấm pin quang điện (tấm pin năng lương mặt trời) dụa trên độ điện dẫn của tấm pin (giải thuật Incremental Conductance Maximum Power Point Tracking - Ind. Cond. MPPT). Đây là phần quan trọng để tạo cơ sở triển khai sủ dụng năng lượng mặt trời trong các ao nuôi, đặc biệt là giải quyết nhu cầu về năng lương cho các dàn quạt nước ao tôm (dùng cho cung cấp ôxy và đảo nước trong ao), vì nó giúp khai thác tối đa nguồn năng lương tù tấm pin năng lượng mặt trời. Dựa trên cơ sở lý thuyết về mô hình của tấm pin quang điện, giải thuật Incremental Conductance được triển khai trong cấu hình mach chuyển đổi điện áp DC-DC kiểu boost xen kẽ đượ điều khiên bởi bộ điều khiển kỹ thuật số khả trình (vi điều khiển STM32F1). Kết quả triển khai cho thấy điện áp tại điểm công suất cực đại của hệ pin được duy trì ổn định, công suất trich xuất được điều khiển bám theo công suất khả dụng tối đa của hệ các tấm pin, hệ thống với giải thuật Ind. Cond. MPPT hoạt động ổn định và không rơi vào vùng thay đổi đặc tính truyền của bộ biến đổi $D C-D C$. 


\section{GIỚI THIẸU}

Trong những năm gần đây, với chính sách thúc đẩy phát triển năng lượng sạch, sự phát triển mạnh mẽ của ngành công nghiệp sản xuất tấm pin năng lượng mặt trời cùng với các tiến bộ về công nghệ điện tử công suất đã thúc đẩy quá trình ứng dụng năng lượng mặt trời vào các lĩnh vực đời sống một cách hiệu quả và rộng rãi trên toàn thế giới. Trong đó, nông nghiệp là một trong các lĩnh vực được ưu tiên quan tâm. Hiện tại, năng lượng mặt trời phục vụ cho nông nghiệp chủ yếu được ứng dụng trong các hệ thống cung cấp nước tưới tiêu, các hệ thống quan trắc và giám sát thông số môi trường,... Lĩnh vực nuôi trồng thủy sản tiêu thụ một lượng năng lượng khá lớn, chủ yếu để cung cấp cho hệ thống quạt nước và cung cấp không khí cho vật nuôi.

Hiện nay, hầu hết các dàn quạt nước trong ao nuôi tôm công nghiệp đều sử dụng động cơ diesel hoặc động cơ điện AC bất đồng bộ. Các động cơ diesel có nhược điểm về hiệu quả làm việc so với giá thành nhiên liệu, hoạt động với tiếng ồn lớn, gây ô nhiễm môi trường nước, môi trường không khí và có chi phí bảo trì cao. Các động cơ điện $\mathrm{AC}$ được ứng dụng rộng rãi hơn do khắc phục được hầu hết các nhược điểm của động cơ diesel. Tuy nhiên, nhược điểm lớn nhất của các động cơ điện $\mathrm{AC}$ là chúng đòi hỏi nguồn điện $\mathrm{AC}$ sằn có, điều này gây trở ngại lớn cho việc triển khai nuôi tôm công nghiệp ở những vùng không sẵn có điện lưới hoặc điện lưới không đủ công suất. Mô hình quạt nước với động cơ $\mathrm{AC}$ chạy bằng năng lượng mặt trời được đề xuất nhằm khắc phục nhược điểm của mô hình quạt nước chạy bằng điện lưới sẵn có, nhằm thúc đẩy sự mở rộng vùng nuôi tôm công nghiệp và không phụ thuộc hóa với lưới điện AC.

Có hai vấn đề chủ yếu cần giải quyết trong hệ thống chuyển đổi năng lượng cho động cơ $\mathrm{AC}$ trong dàn quạt nước: dò tìm và trích xuất được điểm làm việc của hệ các tấm pin quang điện mà tại đó công suất làm việc của tấm pin là tối đa trong một điều kiện môi trường xác định (MPP - Maximum Power Point); và vấn đề cân bằng dòng năng lượng vào ra của hệ thống chuyển đổi năng lượng cho các hệ khai thác năng lượng tái tạo như điện gió và điện mặt trời (cân bằng điện áp chuyển tiếp, trong trường hợp hệ thống sử dụng giải pháp có mạch trích xuất năng lượng tối đa tách biệt với mạch điều khiển tải). Trong các hệ thống sử dụng tải thay đổi, như hệ thống quạt nước, vấn đề khai thác triệt để năng lượng khả dụng từ tấm pin là một trong những vấn đề có tầm quan trọng hàng đầu trong hệ thống. Các phương pháp tối ưu năng lượng khai thác được từ hệ các tấm pin quang điện đều nhằm cố gắng đưa điện áp làm việc về một giá trị mà tại đó công suất phát từ hệ là cực đại trên toàn miền điện áp.

Một số bài báo đề xuất triển khai mô hình dò tìm và bám theo điểm công suất cực đại trong hệ chuyển đổi điện áp DC-DC thông qua việc thay đổi độ rộng xung trực tiếp không thông qua khâu điều chỉnh điện áp như Charais (2010), Nguyễn Ngọc Trung (2012), Nguyễn Văn Tấn và ctv. (2018), Rao et al. (2017) và Vamja \& Mulla (2018). Các mô hình này tuy có đặc điểm đơn giản trong quá trình triển khai nhưng lại dễ bị kẹt vào vùng chuyển giao giữa hai chế độ làm việc của mạch $\mathrm{DC}-\mathrm{DC}$. Tại đó, giải thuật trích xuất không thể đưa hệ thống đến điểm công suất cực đại thật sự của hệ quang điện. Điều này gây ra trở ngại lớn cho người nghiên cứu trong giai đoạn tiếp cận và ứng dụng các giải thuật này.

Để khắc phục các khó khăn hiện có, bài báo tập trung giải quyết vấn đề triển khai giải thuật trích xuất công suất khả dụng tối đa của hệ thống quang điện cỡ nhỏ (công suất đỉnh $1 \mathrm{kWp}$ ) vào hệ chuyển đổi điện áp DC-DC, bao gồm các nội dung chủ yếu: giới thiệu giải thuật Inc. Cond. MPPT, triển khai giải thuật trong hệ thống chuyển đổi điện áp DC-DC, khảo sát đặc tính làm việc thực tế của hệ các tấm pin và một số vấn đề có liên quan.

\section{PHƯƠNG PHÁP NGHIÊN CÚU}

\subsection{Tổng quan}

\subsubsection{Phạm vi ứng dụng của đề tài}

Đề tài này nhắm tới khả năng ứng dụng cho các ao nuôi tôm sử dụng các máy quạt nước. Đối với mô hình nuôi tôm hoặc cá có sử dụng quạt nước để cung cấp oxy, tạo dòng chảy để đảo nước và xi phông đáy, nhu cầu sử dụng năng lượng điện năng dành cho các dàn quạt này là lớn nhất trong số các nhu cầu năng lượng của ao.

Tùy theo quy mô ao nuôi được quy hoạch mà có thể kết hợp sử dụng nhiều hệ quang điện cỡ nhỏ với công suất đỉnh $1 \mathrm{kWp}$ như đã đề cập, với số lượng hệ quang điện chỉ bị giới hạn bởi chi phí, nhu cầu và diện tích lắp đặt. Điều này mang đến khả năng tùy biển về nhu cầu mở rộng hoặc thu hẹp quy mô ao nuôi và tối ưu khả năng trích xuất năng lượng của bộ điều khiển (giảm ảnh hưởng của hiện tượng bóng không đều trên toàn ao).

\subsubsection{Giải thuật dò tìm điểm công suất dụa trên độ điện dẫn của tấm pin quang điện}

a. Đặc tính của tấm pin quang điện

Tế bào quang điện (PV cell), gọi tắt "tế bào", là thành phần cơ bản cấu tạo nên một tấm pin quang 
điện. Một tấm pin quang điện được chế tạo bằng cách phối hợp song song và/hoặc nối tiếp nhiều tế bào quang điện với nhau, đặc tính của tế bào quang điện đại diện cho đặc tính của tấm pin quang điện. Cường độ ánh sáng mặt trời (bức xạ) và nhiệt độ làm việc là các yếu tố ảnh hưởng chính tới các đặc tính hoạt động của các tế bào. Dòng điện của tế bào thay đổi gần như tỷ lệ với cường độ bức xạ nhận được nhưng điện áp chỉ thay đổi nhẹ khi cường độ bức xạ thay đổi. Ngược lại, điện áp chịu sự chi phối mạnh từ yếu tố nhiệt độ, khi nhiệt độ của tế bào tăng cao thì điện áp bị giảm đáng kể, trong khi đó dòng điện chỉ thay đổi một lượng nhỏ. Sự ảnh hưởng của cường độ bức xạ và nhiệt độ lên đặc tính của tấm pin quang điện được mô tả như trong Hình 1.

Sự thay đổi về cường độ ánh sáng có sức ảnh hưởng tới công suất đầu ra của tế bào đáng kể hơn sự thay đổi về nhiệt độ. Điều này đúng cho hầu hết các loại vật liệu sản xuất tế bào thông dụng. Tóm lại,

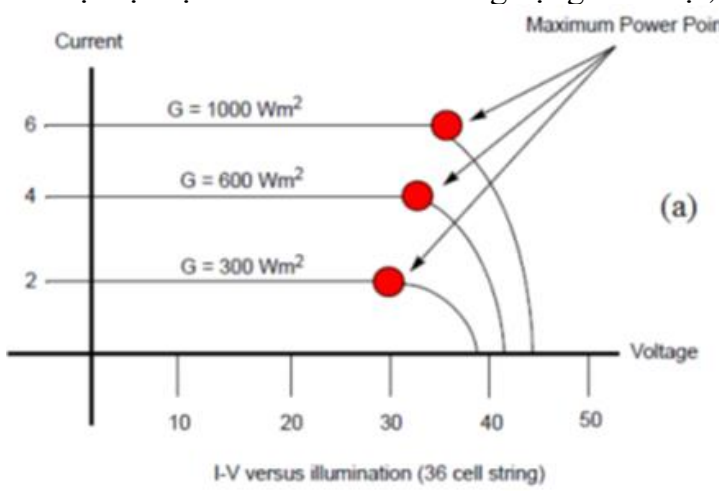

công suất của tấm pin quang điện sẽ bị suy giảm khi cường độ ánh sáng giảm xuống hoặc nhiệt độ của tấm pin tăng lên.

\section{b. Điểm công suất cực đại}

Một tấm pin quang điện có thể hoạt động trong một dải điện áp và dòng điện rộng. Nếu mắc một điện trở vào đầu ra của tấm pin với giá trị tăng từ 0 $\Omega$ (xem như điều kiện ngắn mạch - dòng điện là tối đa) lên tới một giá trị rất lớn (tương đương mạch hở - dòng điện tối thiểu), điểm công suất cực đại có thể được xác định dựa vào đặc tuyến công suất theo dòng điện như trong Hình $2 \mathrm{~b}$. Điểm công suất cực đại là điểm hoạt động của tấm pin mà tại đó tích số giữa dòng điện và điện áp của tấm pin là lớn nhất trong toàn miền điện áp, trong điều kiện các yếu tố môi trường xác định. Công suất đầu ra của tấm pin ở điều kiện ngắn mạch (SC - short circuit) hoặc hở mạch $(\mathrm{OC}-$ open circuit) được xem như bằng $0 \mathrm{~W}$.

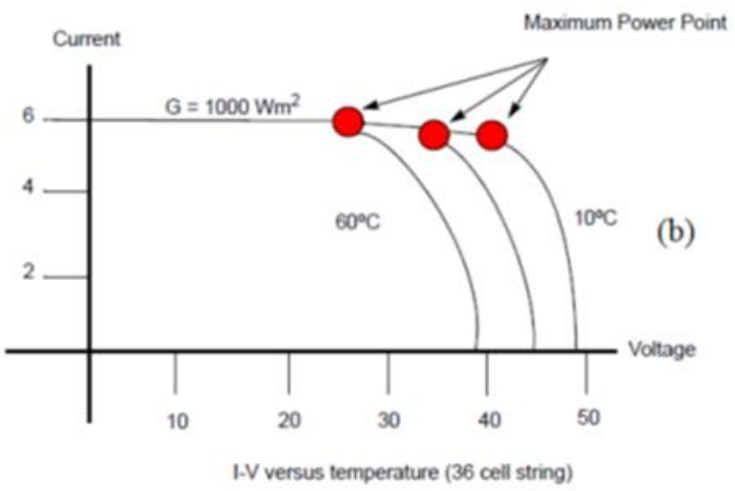

Hình 1. Đặc tính tải của tấm pin quang điện 36 tế bào: (a) ảnh hưởng của cường độ chiếu sáng; (b) ảnh hưởng của nhiệt độ của tấm nền

Hình 2 cho thấy dòng điện và công suất đầu ra của một tấm pin quang điện, trong một điều kiện

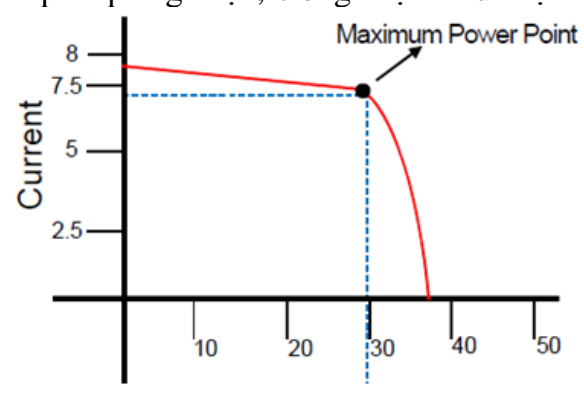

chiếu sáng xác định, như các hàm theo theo điện áp đầu ra (Microchip, 2011).

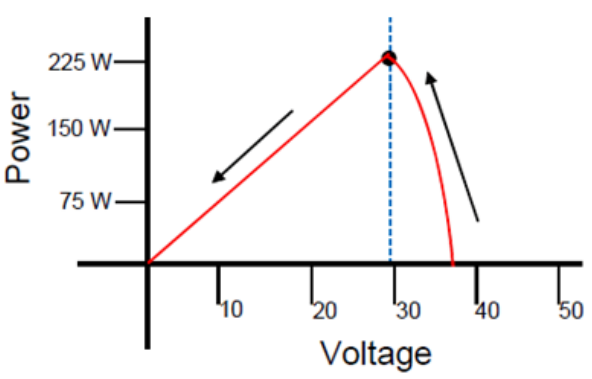

Hình 2. Đặc tính điểm công suất cực đại của một tấm pin quang điện

Hệ thống khai thác năng lượng từ các tấm pin quang điện bắt buộc phải đảm bảo rằng tấm pin luôn hoạt động ở điểm công suất cực đại, để hệ chuyển đổi năng lượng và tải tiêu thụ luôn thu được nhiều năng lượng nhất từ hệ quang điện. Giải thuật MPPT (Maximum Power Point Tracking), chịu trách nhiệm đảm bảo điều kiện này. Độ gợn điện áp trong lúc hoạt động của tấm pin phải đủ nhỏ để giải thuật 
MPPT có thể dò đúng điểm công suất cực đại, nghĩa là tấm pin phải hoạt động với giá trị công suất trung bình tức thời dao động không quá nhiều xung quanh giá trị công suất cực đại thực sự của nó và các tác nhân gây nhiễu phải đủ nhỏ để không ảnh hưởng đến tiến trình MPPT. Các tác nhân gây nhiễu tiêu biểu bao gồm: độ gợn dòng điện, điện áp, hiện tượng ground loop, nhiễu điện từ từ mạch chuyển đổi điện áp DC-DC kiểu switching, và thậm chí là sai số lượng tử đến từ quá trình lấy mẫu/tính toán trong bộ điều khiển.

\section{c. Giải thuật Inc. Cond. MPPT}

Giải thuật Inc. Cond. MPPT dựa vào giả thiết về đặc tuyến công suất theo điện áp của tấm pin. Giả thiết được đặt ra và đã được kiểm chứng là: đồ thị đặc tính của tấm pin tồn tại một cực đại trong toàn miền điện áp với điều kiện các tế bào trên tấm pin được chế tạo đồng chất, không có tình trạng hư hỏng đối với bất kỳ tế bào nào và độ rọi của ánh sáng trên mỗi tế bào là như nhau, tại đó công suất làm việc của tấm pin là tối đa. Vì công suất của tấm pin là hàm khả vi trong mọi điều kiện làm việc, điều này đồng nghĩa với đạo hàm của công suất theo điện áp tại đây bằng 0 . Đây là điểm MPP của tấm pin quang điện.
Hơn nữa, đạo hàm của công suất theo điện áp mang giá trị dương khi tấm pin hoạt động tại điểm công suất ở bên trái điểm MPP và mang giá trị âm khi tấm pin hoạt động tại điểm công suất ở bên phải điểm MPP. Phương trình (1) biểu diễn ý tưởng này. Trong đó, $\mathrm{dP}$ là độ dời công suất và $\mathrm{dV}$ là độ dời điện áp.

$$
\begin{aligned}
& \frac{\mathrm{dP}}{\mathrm{dV}}=0 \text { tại MPP, } \\
& \frac{\mathrm{dP}}{\mathrm{dV}}>0 \text { bên trái MPP, } \\
& \frac{\mathrm{dP}}{\mathrm{dV}}<0 \text { bên phải MPP }
\end{aligned}
$$

Giải thuật Inc. Cond. MPPT dựa vào giá trị đạo hàm công suất theo điện áp làm việc của tấm pin để thay đổi điện áp tham chiếu đưa tới bộ điều khiển điện áp tấm pin. Lưu đồ giải thuật trong Hình 3 diễn giải chi tiết cho giải thuật này, với $\mathrm{V}[\mathrm{k}], \mathrm{I}[\mathrm{k}]$ và $\mathrm{P}[\mathrm{k}]$ lần lượt là điện áp, dòng điện và công suất làm việc tại lần đo gần nhất và $\mathrm{V}[\mathrm{k}-1], \mathrm{I}[\mathrm{k}-1]$ và $\mathrm{P}[\mathrm{k}-1]$ lần lượt là điện áp, dòng điện và công suất làm việc tại lần đo liền kề trước lần đo hiện tại.

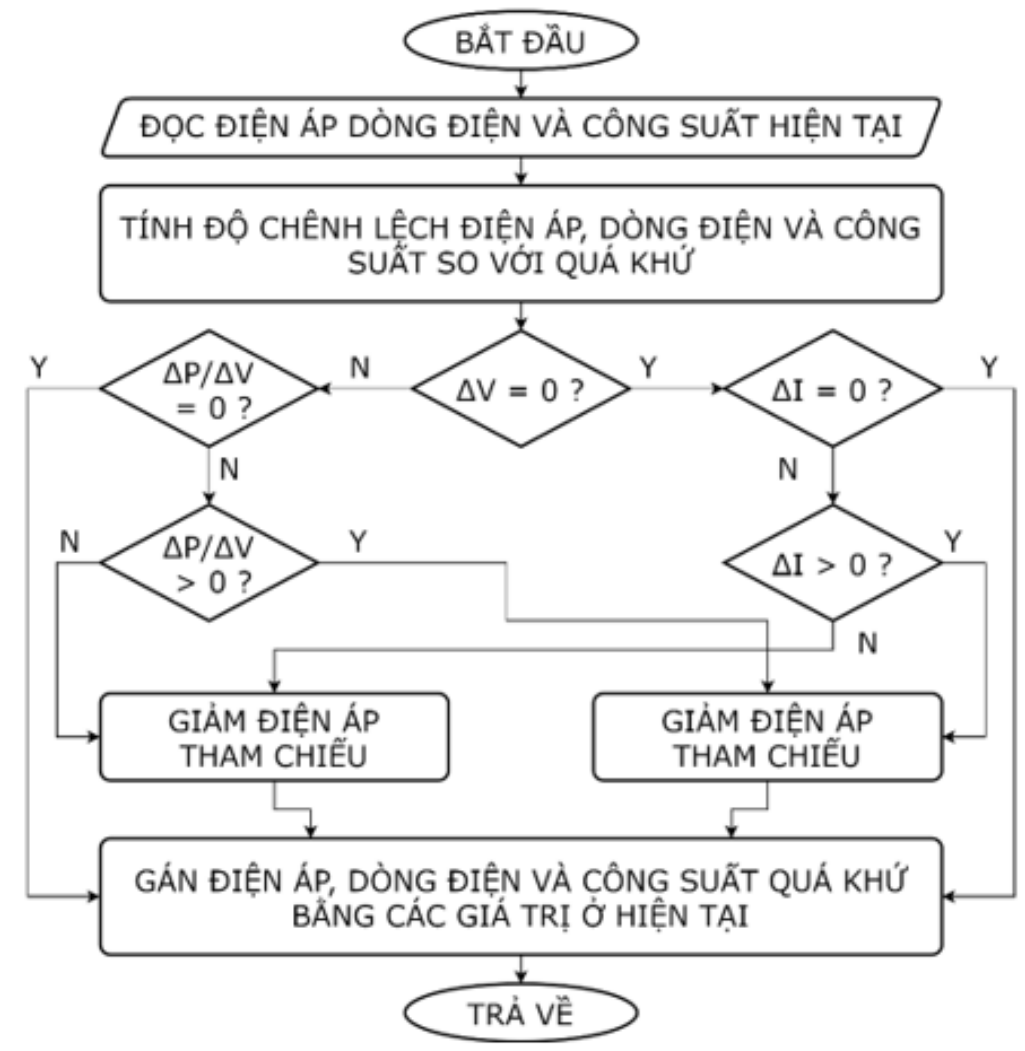

Hình 3. Lưu đồ của giải thuật Inc. Cond. MPPT 
Giải thuật Inc. Cond. MPPT có hai nhược điểm chính: không thể phát hiện chính xác hiện tượng nửa bóng và nhạy cảm với nhiễu. Hiện tượng nửa bóng là hiện tượng các tấm pin trong hệ quang điện không nhận được mức bức xạ đều nhau. Trong điều kiện này, đồ thị công suất theo điện áp của tấm pin có nhiều hơn một cực đại, điều này có thể làm cho điểm cực đại được giải thuật phát hiện có khả năng không phải là cực đại lớn nhất trên toàn miền (do giả thuyết đồ thị công suất chỉ có một điểm cực đại không còn đúng nữa). Ngoài ra, do tồn tại toán tử vi phân, giải thuật này nhạy cảm với nhiễu vì bản chất của vi phân là bộ lọc thượng thông, điều này đòi hỏi tín hiệu đầu vào của bộ dò phải có tỷ lệ nhiễu thấp, đồng nghĩa với việc yêu cầu các bước xử lý nhiễu cả trên phần cứng và phần mềm. Tuy nhiên, ưu điểm của giải thuật này so với các phương pháp đơn giản khác (giải thuật Perturb and Observe, phương pháp ước lượng điện áp MPP...) là nó có thể dò điểm công suất cực đại một cách chính xác và ít dao động xung quanh điểm này, góp phần giảm hao phí trong quá trình trích xuất năng lượng cũng như tăng chất lượng điện đầu ra.

\subsection{Triển khai giải thuật với mạch chuyển đổi DC-DC kiểu boost xen kẽ}

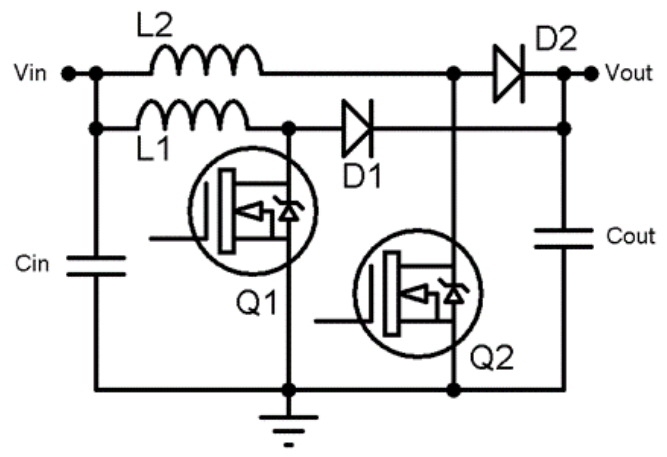

\section{Hình 4. Sơ đồ nguyên lý đơn giản của mạch boost xen kẽ}

Kiểu cấu hình mạch boost xen kẽ có cấu tạo tương tự như hai mạch boost đấu song song đầu vào và đầu ra, tuy nhiên, tín hiệu điều khiển của các van công suất có pha lệch nhau $180^{\circ}$ trong một chu kỳ. Do đặc tính nghịch pha của tín hiệu điều khiển nên hai van công suất cũng đóng mở nghịch pha tương ứng, điều này góp phần tạo nên các ưu điểm chính của kiểu mạch này: giảm độ gợn dòng đầu vào và giảm độ gợn áp đầu ra. Hình 4 mô tả sơ đồ nguyên lý của kiểu cấu hình mạch này.

Độ gợn dòng điện đầu vào được xem như bằng 0 nếu độ rộng của hai xung điều khiển bằng $50 \%$, trong các ứng dụng đòi hỏi độ tin cậy và hệ thống hoạt động bền bỉ, điều này trở nên rất quan trọng. Tuổi thọ của các tụ điện hóa trong hệ thống chuyển đổi DC-DC giảm đáng kể nếu chúng phải thường xuyên hoạt động với độ gợn dòng cao. Thêm vào đó, do trong một chu kỳ đóng ngắt có hai xung điều khiển van bán dẫn nên tần số dòng điện nạp tụ Cout bằng gấp đôi tần số điều khiển van công suất. Điều này làm cho độ gợn điện áp đầu ra của mạch giảm gấp đôi.

Tương tự như kiểu mạch boost thường, kiểu mạch boost xen kẽ cũng có hai chế độ làm việc: chế độ dòng liên tục và chế độ dòng không liên tục, tương ứng với độ liên tục của dòng điện đi qua cuộn cảm. Tải đầu ra và giá trị điện cảm quyết định chế độ làm việc của mạch. Tại chế độ dòng không liên tục, dòng điện của cuộn cảm giảm xuống 0 trước khi bắt đầu chu kỳ mới. Trong chế độ này, hàm truyền điện áp theo độ rộng xung của mạch có dạng phức tạp và khó ước lượng. Tại chế độ dòng liên tục, dòng điện đi qua các cuộn cảm luôn lớn hơn 0 . Hàm truyền của mạch trong chế độ này chỉ phụ thuộc vào độ rộng xung như trong phương trình (2), trong đó $\mathrm{V}_{\text {out }}, \mathrm{V}_{\text {in }}$ và $\mathrm{D}$ lần lượt là biên độ điện áp đầu ra, biên độ điện áp đầu vào và độ rộng xung của tín hiệu điều khiển MOSFET. Chế độ này còn có ưu điểm là dòng điện đỉnh đi qua các van công suất nhỏ hơn (Kumar, R., Singh, B., Chandra, A. and Al-Haddad, K., 2015), nhằm làm giảm chi phí cho linh kiện bán dẫn, dễ dàng ứng dụng cho các bộ chuyển đồi có mật độ công suất/thể tích lớn và tạo ra ít nhiễu điện từ hơn. Vì những ưu điểm kể trên, trong bài báo này, các thành phần linh kiện được thiết kế theo khoảng tải sao cho mạch boost xen kẽ luôn chạy ở chế độ dòng liên tục.

$$
\mathrm{V}_{\text {out }}=\frac{\mathrm{V}_{\text {in }}}{1-\mathrm{D}} .
$$

Bài viết này không tập trung phân tích sâu về kiểu mạch boost xen kẽ, thông tin về đặc tính của mạch cũng như các tính toán cần thiết có thể tìm thấy trong các công trình liên quan (Ajit, 2014; Chitra \& Seyezhai, 2014; Coruh et al., 2011; Crews, 2013; Gunawan, 2009; Stasi, 2015; Zdanowski et al., 2017).

Một trong những vấn đề quan trọng hàng đầu khi triển khai giải thuật MPPT với kiểu mạch boost hoặc buck là hàm truyền của mạch có thể thay đổi khi tải đầu ra thay đổi. Về bản chất của mạch boost, khi tải có đặc tính thay đổi công suất cùng chiều với sự thay đổi điện áp, dòng điện đầu vào nhìn chung sẽ tăng khi độ rộng xung tăng, tuy nhiên, độ rộng xung 
không nên được thay đổi trực tiếp trong tiến trình dò tìm điểm công suất. Trong lưu đồ ở Hình 3 , nếu chỉ đơn thuần thay đổi độ rộng xung thay vì thay đổi điện áp tham chiếu trong quá trình dò tìm MPP, hệ thống có nguy cơ rơi vào vùng chuyển giao giữa hai chế độ làm việc của mạch boost. Tại điểm này, điện áp đầu ra thay đổi đột ngột theo hàm truyền, trong trường hợp tải đầu ra là điện trở có giá trị không thay đổi đủ nhanh, dòng điện đầu ra cũng thay đổi theo, kéo theo sự thay đổi đột ngột của dòng điện đầu vào (lúc này độ rộng xung không kịp thay đổi, do tốc độ dò của MPPT tương đối thấp so với những sự thay đổi đã nêu) làm cho giải thuật MPPT hiểu nhầm điểm này là điểm công suất cực đại và bị kẹt tại đó. Điều này được phân tích kỹ trong Oprea et al. (2014).

Vì lý do nêu trên, hệ thống cần có thêm một tiến trình điều khiển có tốc độ đủ nhanh để đưa điện áp làm việc của hệ quang điện về đúng điện áp tham chiếu cho ra bởi tiển trình MPPT. Trong bài báo này, mô hình điều khiển trích xuất công suất khả dụng tối đa của hệ quang điện bao gồm một bộ điều khiền PI kết hợp với giải thuật Inc. Cond. MPPT được đề xuất như trong Hình 5. Trong đó, $\mathrm{Vpv}, \mathrm{Ipv}, \mathrm{Ppv}$ lần lượt là điện áp, dòng điện và công suất của hệ quang điện và $\mathrm{D}$ là tín hiệu điều khiển (độ rộng xung) cho bộ boost. Bộ tích phân trong mô hình đóng vai trò như một bộ lọc hạ thông giúp ổn định tín hiệu trước khi đưa vào bộ dò MPPT. Mô hình này có khả năng hoạt động không phụ thuộc kiểu mạch chuyển đổi DCDC, như đã nêu trong Rosu-Hamzescu et al. (2013).

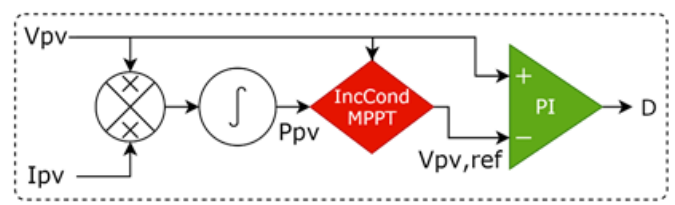

\section{Hình 5. Mô hình điều khiển mạch DC-DC đề xuất}

Trong mô hình nêu trên, Vpv,ref là tín hiệu điện áp MPP tham chiếu được dò bởi giải thuật MPPT. Tín hiệu này là đầu vào đảo (điểm đặt) của bộ điều khiển PI và tín hiệu điện áp đọc về từ hệ quang điện là đầu vào không đảo (tín hiệu hồi tiếp) của bộ PI, sở dĩ điểm đặt và tín hiệu hồi tiếp hoán đổi vị trí cho nhau so với một hệ điều khiển thông thường là vì khi độ rộng xung của mạch càng tăng thì điện áp đầu ra càng giảm, và ngược lại. Để điều khiển điện áp trên hệ quang điện một cách hiệu quả, băng thông của khâu bù PI cần thỏa mãn các yêu cầu sau: cao hơn băng thông của bộ dò MPPT ít nhất 30 lần và đủ nhanh để đáp ứng với đặc tính nạp xả của các linh kiện tích trữ năng lượng trong mạch boost.

Một đặc điểm quan trọng nữa đối với giải thuật Inc. Cond. MPPT là khi hệ thống DC-DC kiểu boost làm việc ở miền điện áp cao hơn điện áp hở mạch của hệ quang điện, giải thuật có thể ngưng dò điểm công suất (bị treo). Nguyên nhân là khi ở vùng này, độ dời điện áp, độ dời dòng điện và độ dời công suất đều bằng 0 , lúc này động rộng xung đầu ra của khâu bù $\mathrm{PI}$ hiển nhiên bằng 0 . Vì vậy, triển khai vào hệ khả trình, điện áp hở mạch của hệ quang điện cần được nhận dạng trước khi giải thuật bắt đầu dò điểm MPP.

Hình 6 minh họa sơ đồ các khối chức năng của mô hình đề xuất. Trong đó, tải đầu ra của bộ boost là bóng đèn sợi đốt có độ thay đổi điện trở không đáng kể so với độ thay đổi điện áp nguồn, có thể xem như điện trở thuần.

\subsection{Triển khai và thu thập dữ liệu}

\subsubsection{Triển khai hệ quang điện và mạch $D C$ - DC}

Hệ quang điện, bao gồm 03 tấm pin có tên mã WE350TU-36MD (WorldEnergy) đấu nối tiếp, được triển khai trên mái nhà, kết quả triển khai được thể hiện qua Hình 8.

Từ các đặc tính của tấm pin quang điện trong Hình 7 , có thể ước lượng điện áp, dòng điện và công suất của hệ quang điện qua các phương trình từ (3) đến (7). Trong đó, STC là điều kiện môi trường trong phòng thí nghiệm và NOCT là điều kiện làm việc ở môi trường thực tế và 0,93 là hệ số suy giảm ước lượng thông qua thực nghiệm trên hệ thống.

$$
\begin{aligned}
& \mathrm{V}_{\text {array,NOCT,OC }}=3 * 0.93 * \mathrm{~V}_{\text {panel,STC,OC }}=123.7365 \mathrm{~V} . \\
& \mathrm{V}_{\text {array,NOCT,MPP }}=3 * 0.93 * \mathrm{~V}_{\text {panel,STC,MPP }}=111.9348 \mathrm{~V} . \\
& \mathrm{I}_{\text {array,NOCT,SC }}=0.93 * \mathrm{I}_{\text {panel,STC,SC }}=8.6862 \mathrm{~A} . \\
& \mathrm{I}_{\text {array,NOCT,MPP }}=0.93 * \mathrm{I}_{\text {panel,STC,MPP }}=8.1189 \mathrm{~A} . \\
& \mathrm{P}_{\text {array,NOCT,MPP }}=\mathrm{V}_{\text {array,NOTC,MPP }} \mathrm{I}_{\text {array,NOTC,MPP }}=908.7874477 \mathrm{~W} .
\end{aligned}
$$




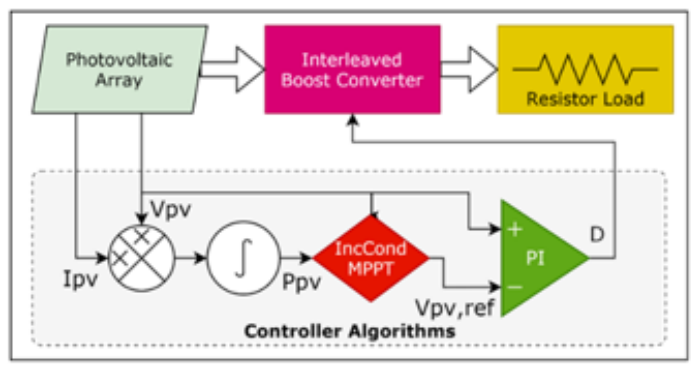

Hình 6. Mô hình chức năng của hệ thống

Mạch biến đổi DC-DC với giải thuật MPPT được triển khai dưới dạng $\mathrm{PCB}$ với các thành phần được chú giải trong Hình 9. Tổ hợp tải bóng đèn sợi đốt được đấu nối để tiêu thụ công suất tương đương $1115 \mathrm{~W}$ tại $340 \mathrm{~V}$, đảm bảo có thể chịu đựng được công suất khả dụng tối đa của hệ quang điện.

\begin{tabular}{|lcc||}
\hline MODULE TYPE: & \multicolumn{2}{c|}{ WE350TU-36MD } \\
\hline Peak Power (Pmax) & (W): & 350 \\
Production Tolerance & $(\%):$ & $0^{2}+3$ \\
Maximum Power Current (Imp) & $(\mathrm{A}):$ & 8.73 \\
Maximum Power Voltage (Vmp) & $(\mathrm{V}):$ & 40.12 \\
Short Circuit Current (Isc) & $(\mathrm{A}):$ & 9.34 \\
Open Circuit Voltage (Voc) & $(\mathrm{V}):$ & 44.35 \\
\hline Weight & $(\mathrm{Kg}):$ & 22.5 \\
Dimensions & $(\mathrm{mm}):$ & $1956^{*} 992^{*} 40$ \\
Maximum System Voltage & $(\mathrm{VDC}):$ & 1000 \\
Application class & $:$ & $\mathrm{A}$ \\
Fire safety class & C & $\mathrm{C}$ \\
Mechanical load tested & $(\mathrm{Pa}):$ & 2400 \\
\hline
\end{tabular}

Hình 7. Đặc tính kỹ thuật của tấm pin quang điện



Hình 8. Hệ thống quang điện được triển khai

\subsubsection{Triển khai giải thuật MPPT và bộ điều khiển điện áp PI}

Giải thuật MPPT và bộ điều khiển điện áp PI được triển khai trong hệ điều khiển kỹ thuật số khả trình, sử dụng vi điều khiển STM32F103C8. Vi điều khiển này có một số thông số nổi bật như sau:
- Tốc độ tính toán tối đa 72 MIPS cho các lệnh cộng trừ và nhân 32 bit.

- Hỗ trợ 02 module timer với khả năng tạo xung PWM lệch pha $180^{\circ}, 02$ module timer thông thường và một system timer. device.

- Có sẵn bộ điều khiển giao tiếp USB kiểu

- Tích hợp vòng khóa pha PLL cho hệ thống xung nhịp nên chỉ yêu cầu thạch anh ngoài với tần số tối đa $8 \mathrm{MHz}$.

- Có sẵn $64 \mathrm{kB}$ bộ nhớ chương trình và $20 \mathrm{kB}$ bộ nhớ RAM.

- Được trang bị 02 module ADC 12 bit với tốc độ lấy mẫu tối đa $1 \mathrm{MSa} / \mathrm{s}$.

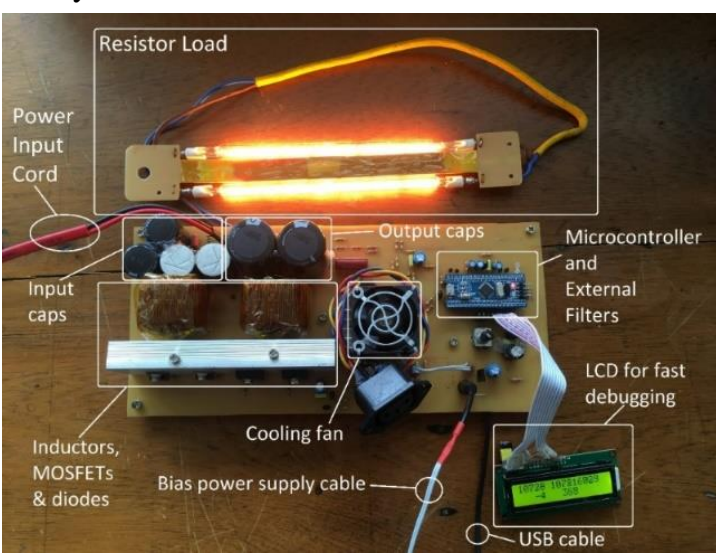

Hình 9. Triển khai phần cứng của hệ thống MPPT

Tần suất của tiến trình Inc. Cond. MPPT được chọn bằng $281,25 \mathrm{~s}^{-1}$ và của tiến trình điều khiển điện áp tấm pin được chọn bằng $36.000 \mathrm{~s}^{-1}$.

Do lõi xử lý Cortex ${ }^{\circledR}$ M3 trong vi điều khiển STM32F103C8 không hỗ trợ kiểu số dấu chấm động nên các kiểu dấu chấm tĩnh được sử dụng phối hợp nhằm tạo độ chính xác cao nhất trong khi vẫn giữ tốc độ tính toán tối đa. Tuy nhiên, nhược điểm của sự phối hợp các kiểu dữ liệu này là chương trình cần phải được chỉnh sửa nhiều nếu tái sử dụng trên hệ thống khác.

Bộ điều khiển PI được rời rạc hóa như sau:

$$
\mathrm{U}[\mathrm{k}]=\mathrm{U}[\mathrm{k}-1]+\mathrm{Kp} * \mathrm{E}[\mathrm{k}]+\mathrm{Ki} *[\mathrm{k}] \text {. }
$$

Trong đó, $\mathrm{I}[\mathrm{k}]$ là thành phần tích phân. Trong bộ điều khiển, thành phần này giúp loại bỏ sai số xác lập (chủ yếu gây ra bởi sai số lượng tử trong hệ thống có lấy mẫu và tính toán kỹ thuật số), được tính như sau: 


$$
\mathrm{I}[\mathrm{k}]=\mathrm{I}[\mathrm{k}]+\mathrm{E}[\mathrm{k}] .
$$

Một lưu ý quan trọng đối với thành phần tích phân là giá trị của nó phải được giới hạn trong một khoảng cố định cho trước, lệnh giới hạn được thực hiện tự động hoặc bằng các lệnh của người lập trình tùy theo khả năng tính toán của CPU. Thông thường, các CPU với tập lệnh DSP sẽ hỗ trợ các lệnh tính toán và giới hạn tự động. Việc giới hạn thành phần tích phân nhằm tránh hiện tượng tràn kiểu dữ liệu trong hệ điều khiển số khả trình (hiện tượng này chủ yếu gây ra do giới hạn của cơ cấu chấp hành, ví dụ, độ rộng xung tối đa cho phép của bộ boost - khoảng $78 \%)$.

Trong Hình 10 là lưu đồ giải thuật của toàn bộ chương trình được triển khai trên vi điều khiển. Lưu đồ đầu tiên bên trái là của chương trình chính, lưu đồ đầu tiên bên phải là của bộ điều khiển PI. Lưu đồ phía trên ở giữa là của tiến trình phục vụ gửi dữ liệu lên máy vi tính. Lưu đồ còn lại là của tiến trình dò tìm điện áp tại MPP, trong đó, tiến trình con TÍNH TOÁN INC. COND. có lưu đồ giải thuật như đã trình bày trong Hình 3 .



Hình 10. Lưu đồ của các tiến trình chính trong mô hình 


\subsubsection{Thu thập dũ liệu qua ưng dụng SerialPlot}

Dữ liệu từ mô hình phần cứng được thu thập và vẽ đồ thị minh họa bằng ứng dụng SerialPlot với tốc độ $1 \mathrm{kSa} / \mathrm{s}$. SerialPlot là ứng dụng hỗ trợ thu thập dũ liệu, vẽ đồ thị theo thời gian và kết xuất thành tệp csv trên cơ sở dữ liệu thu thập được thông qua giao thức truyền thông nối tiếp Serial. Ứng dụng này có một số đặc điểm nổi bật như sau:

- Là phần mềm mã nguồn mở, tương thích với hệ điều hành Windows ${ }^{\circledR}$ và Linux ${ }^{\circledR}$.

- Hỗ trợ tối đa 32 kênh tín hiệu với các kiểu số uint 8 , uint 16 , uint 32 , int 8 , int 16 , int 32 và float.
- Hỗ trợ cả hai loại Endianness.

- Có chức năng kết xuất dữ liệu thành tệp csv.

Đường truyền thông từ vi điều khiển STM32F103 và máy vi tính được thiết lập qua giao thức USB. Vi điều khiển kết nối với máy vi tính thông qua kết nối USB kiểu giả lập cổng truyền thông nối tiếp ảo, hỗ trợ tốc độ baudrate tối đa hơn $1 \mathrm{MBd}$.

Hình 11 minh họa giao diện vẽ đồ thị của ứng dụng SerialPlot với giá trị các biến số đã được nhân với 100 (nhằm tăng độ phân giải).

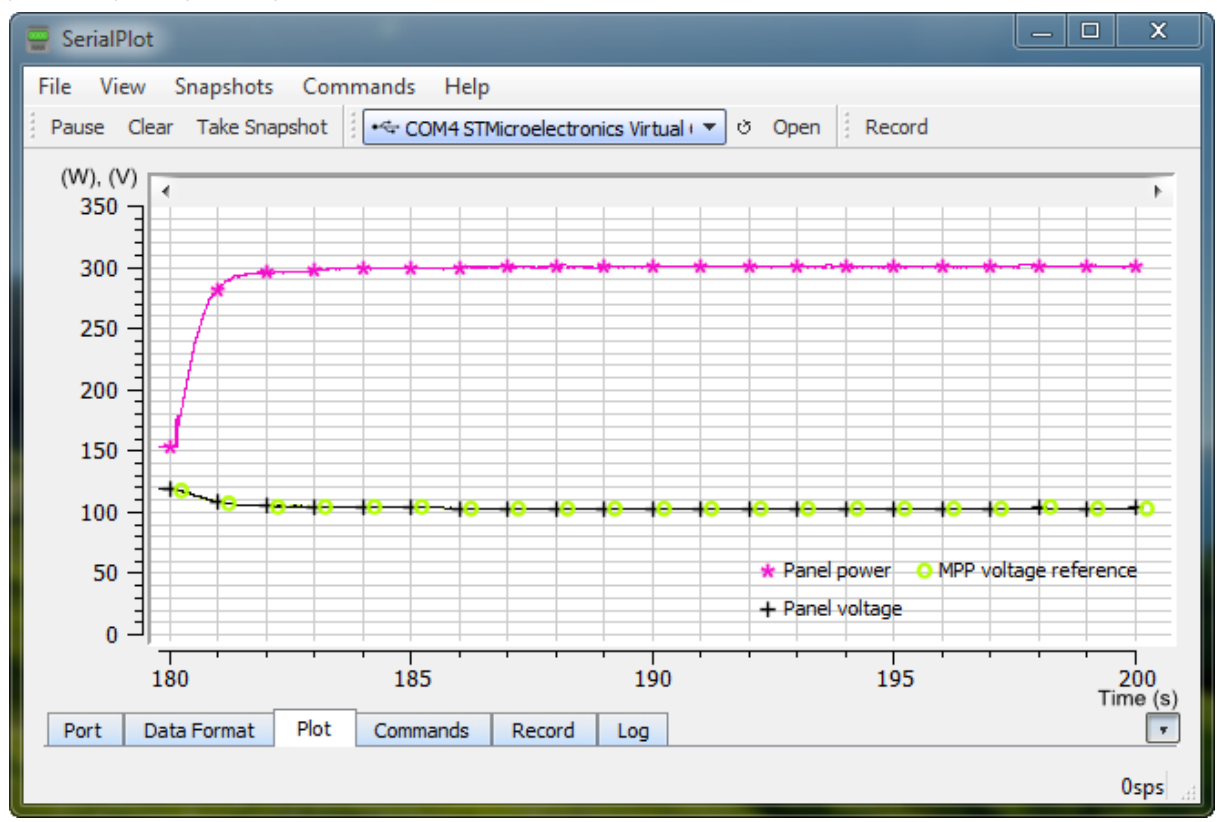

Hình 11. Giao diện chính của ứng dụng SerialPlot

\section{KÊT QUẢ VÀ THẢO LUẬN}

\section{1. Độ ổn định công suất và điện áp của hệ pin}

Hình 12 thể hiện đáp ứng khởi động và làm việc của giải thuật Inc. Cond. MPPT với cường độ bức xạ $300 \mathrm{~W} / \mathrm{m}^{2}$. Tại giây thứ 180 , hệ thống bắt đầu hoạt động, điện áp tham chiếu được cài trước bằng 98\% điện áp hở mạch của hệ quang điện, bộ dò MPPT và bộ điều khiển PI được cho phép hoạt động, mạch boost bắt đầu bám theo điểm công suất cực đại của hệ quang điện. Công suất trích xuất xác lập sau thời gian 1,5 giây. Từ điểm này đến 200 giây, điện áp hoạt động của hệ quang điện được ổn định tại điểm xấp xỉ 110,3 V; công suất đầu ra của hệ quang điện được ổn định tại điểm xấp xỉ 300 W.
Hình 13 thể hiện đáp ứng khởi động và làm việc của giải thuật Inc. Cond. MPPT với cường độ bức xạ $250 \mathrm{~W} / \mathrm{m}^{2}$. Công suất trích xuất xác lập sau thời gian 4 giây. Từ điểm này đến 200 giây, điện áp hoạt động của hệ quang điện được ổn định tại điểm xấp xỉ 110,3 V; công suất đầu ra của hệ quang điện được ổn định tại điểm xấp xỉ $248 \mathrm{~W}$.

Điều này chứng tỏ giải thuật MPPT hoạt động ổn định nhiễu và điện áp đầu ra của hệ quang điện được khống chế tại MPP.

\section{2. Đáp ứng của khâu bù điện áp $P I$}

Đáp ứng của khâu bù điện áp PI tại các điểm làm việc $300 \mathrm{~W}$ và $248 \mathrm{~W}$ lần lượt được minh họa trong Hình 14 và Hình 15 . Có thể thấy, kể từ thời điểm cho phép dò MPP, tín hiệu đầu ra (đường màu đen) bám rất tốt theo tín hiệu đặt (đường màu xanh). 


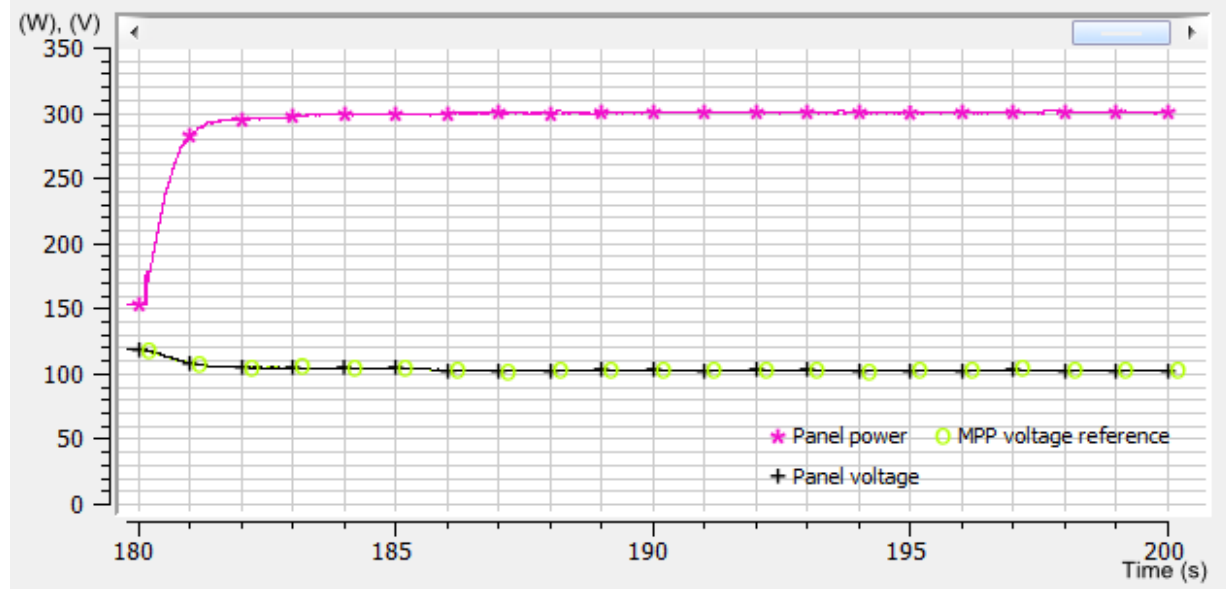

Hình 12. Độ ổn định điện áp và công suất tại $300 \mathrm{~W}$

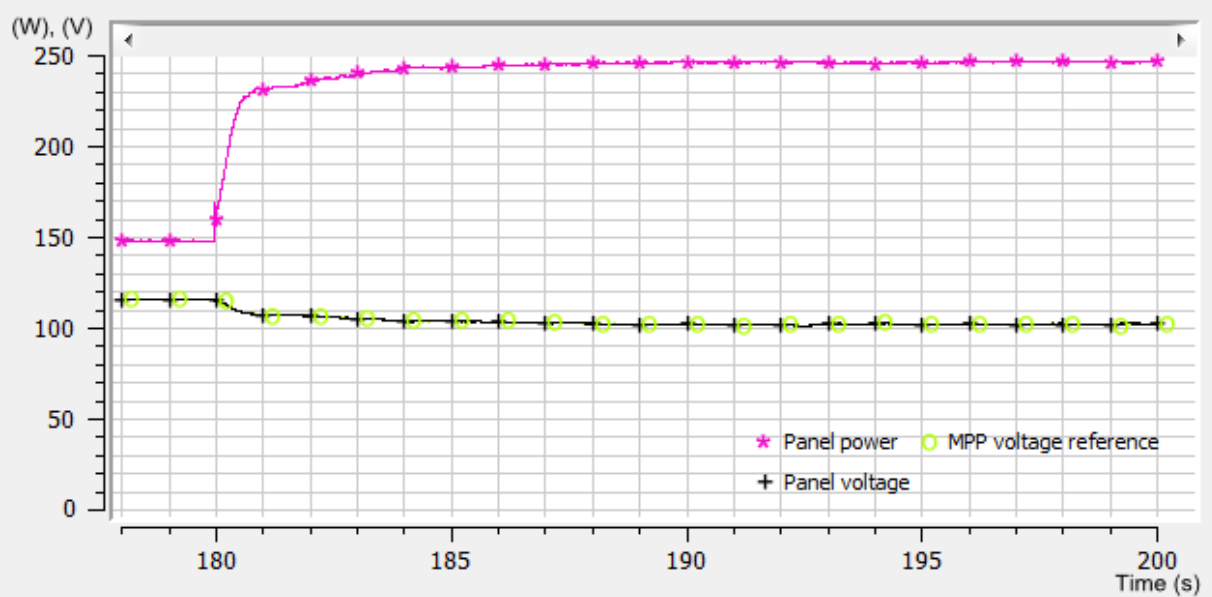

Hình 13. Độ ổn định điện áp và công suất tại $248 \mathrm{~W}$

\section{KẾT LUẬN VÀ ĐỀ XUẤT}

\subsection{Kết luận}

Bài báo đã đề xuất, phân tích và triển khai thành một mô hình dò tìm điểm công suất cực đại dựa trên giải thuật Inc. Cond MPPT và bộ chuyển đổi điện áp DC-DC kiểu boost xen kẽ, sử dụng vi điều khiển STM32F103C8. Mô hình hoạt động ổn định trong dải công suất dưới $300 \mathrm{~W}$. Trong hai trường hợp công suất bức xạ bằng $300 \mathrm{~W}$ và $250 \mathrm{~W}$, độ bám của giải thuật Inc. Cond. ổn định và không có dao động. Đặc biệt, đặc tính bám điểm đặt của khâu bù điện áp có thể được đánh giá là rất tốt.

\section{2. Đề xuất hướng nghiên cứu}

Bài báo này là cơ sở để phát triển các hệ thống chuyển đổi năng lượng dùng trong các lĩnh vực năng lượng, đặc biệt là hệ thống dùng cho tải thay đồi trong lĩnh vực nông nghiệp như hệ thống tổ hợp máy quạt ao tôm, hệ thống tích trữ nước thủy lợi, các hệ thống quan trắc, thu thập dữ liệu và điều khiển thông số môi trường trong nông nghiệp thông minh.

Cụ thể, các máy quạt ao tôm chạy bằng động cơ điện $\mathrm{AC}$ không đồng bộ, có thể hoạt động từ nguồn điện DC thông qua biến tần (inverter). Một inverter kết hợp với động cơ $\mathrm{AC}$ có thể được xem là tải $\mathrm{DC}$ có thể dễ dàng điều khiển thay đổi nội trở (thông qua các tham số như biên độ điện áp và tần số dòng điện điều chế). Hệ thống hợp thành từ inverter và bộ chuyển đổi $\mathrm{DC}-\mathrm{DC}$ là một hướng nghiên cứu rất tiềm năng. Trong kịch bản này, bộ chuyển đổi DCDC với giải thuật MPPT sẽ có vai trò trích xuất nguồn năng lượng tối đa từ các tấm pin mặt trời và bộ inverter với giải thuật cân bằng công suất vào/ra sẽ có vai trò điều chế dòng điện $\mathrm{AC}$ làm quay động cơ kéo cánh quạt với công suất tối đa được trích xuất từ các tấm pin. 


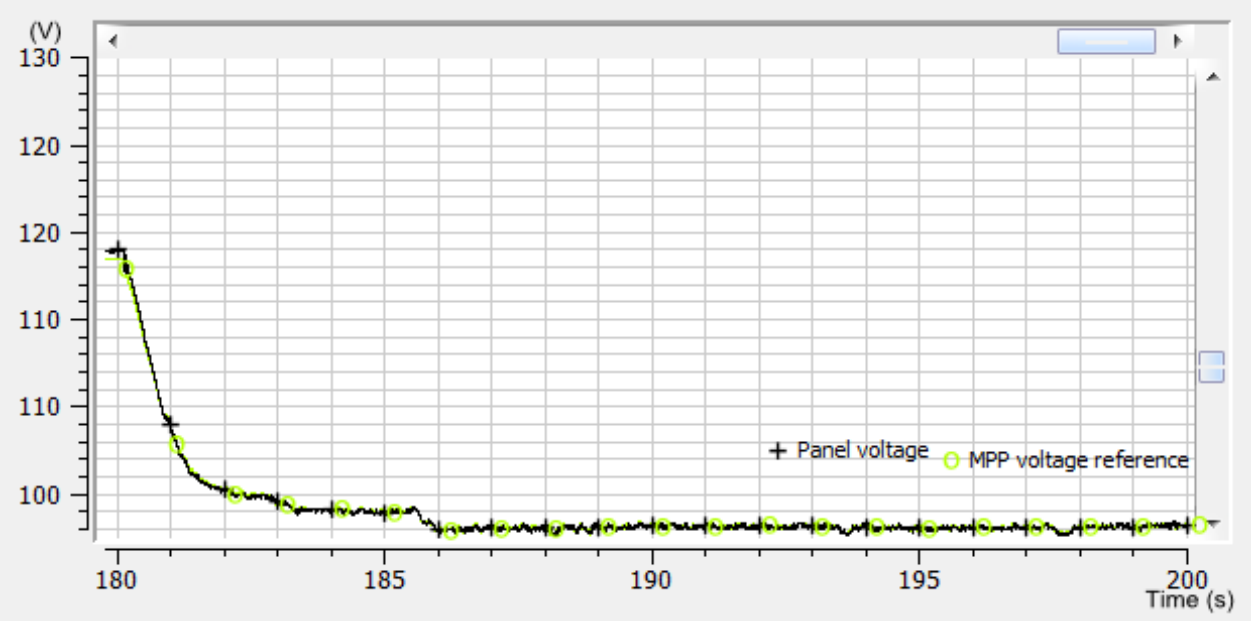

Hình 14. Đáp ứng của bộ điều khiển điện áp tại $300 \mathrm{~W}$

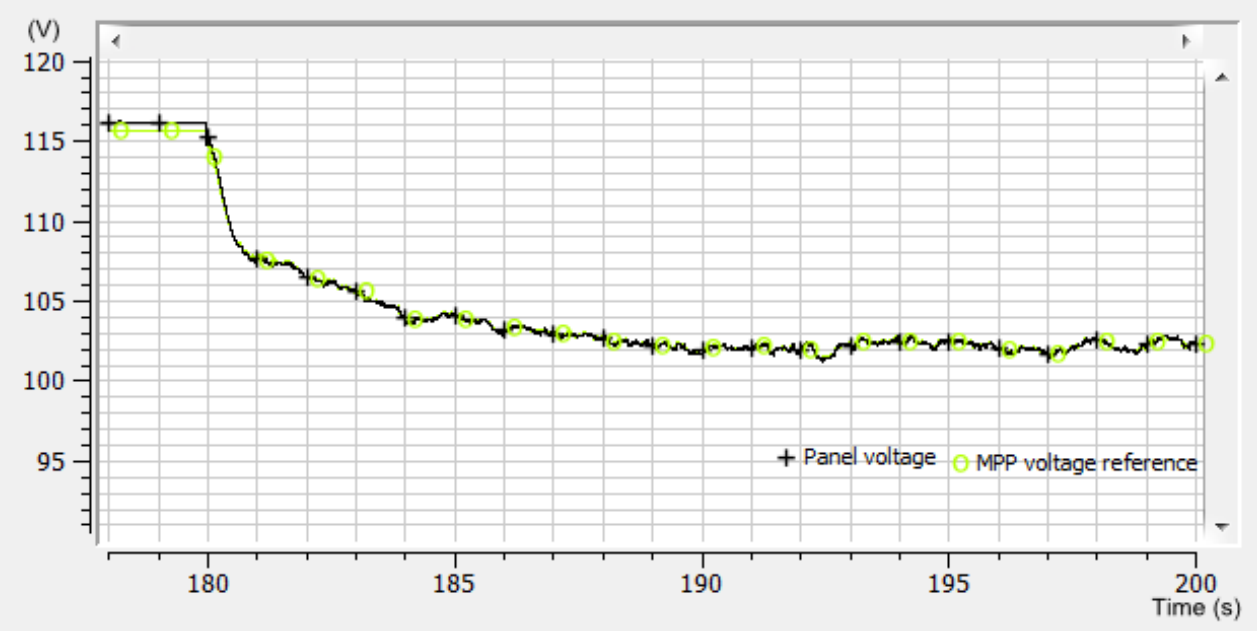

Hình 15. Đáp ứng của bộ điều khiển điện áp tại $248 \mathrm{~W}$

Trong tương lai, hướng mở rộng chủ yếu của mô hình trích xuất năng lượng này là tích hợp thêm các giải thuật dò tìm MPP cho hệ quang điện có quy mô lớn mà tại đó, vấn đề nửa bóng gây ảnh hưởng đáng kể tới hiệu quả hoạt động của toàn hệ thống, để cấu thành nên các hệ thống lớn hơn.

Hiện tại, có ba hệ thống chuyển đổi năng lượng phù hợp đẻ ứng dụng mô hình trích xuất năng lượng trong nghiên cứu này:

- Hệ thống tích trữ năng lượng: có thể được triển khai dễ dàng dựa trên mô hình này, mô hình tích trữ này chỉ đòi hỏi thêm các tiến trình bảo vệ và khống chế công suất tối đa nạp vào thiết bị lưu trữ.

- Hệ thống chuyển đổi năng lượng cho phụ tải có khả năng thay đồi: đòi hỏi phải có tiến trình cân bằng công suất vào ra của hệ thống (công suất làm việc tối đa của hệ pin và công suất tác dụng của tải) và tiến trình khống chế công suất.

- Hệ thống hòa lưới: khi xem lưới điện là tải tiêu thụ, hệ thống này được xem như hệ thống chuyển đổi năng lượng cho phụ tải có khả năng thay đổi.

\section{LỜI CÁM ƠN}

Nghiên cứu này được tài trợ một phần từ Dự án nâng cấp trường Đại học Cần Thơ VN14-P6 được hỗ trợ bởi nguồn vốn ODA của Chính phủ Nhật Bản.

\section{TÀI LIỆU THAM KHẢO}

Ajit, T. N. (2014). Two Stage Interleaved Boost Converter Design and Simulation in CCM and DCM. India International Journal of Engineering Research \& Technology (IJERT) Vol, 847-851. 
Charais, J. (2010). Maximum Power Solar Converter (Application Note No. DS01211B). Retrieved October 9, 2020, from Microchip website: http://ww1.microchip.com/downloads/en/AppNo tes/01211B.pdf.

Chitra, P., \& Seyezhai, R. (2014). Basic design and review of two phase and three phase interleaved boost converter for renewable energy systems. International journal of applied science, 1(1), 1-26.

Coruh, N., Urgun, S., Erfidan, T., \& Ozturk, S. (2011, June). A simple and efficient implemantation of interleaved boost converter. In 2011 6th IEEE Conference on Industrial Electronics and Applications (pp. 2364-2368). IEEE.

Crews, R. (2013). AN-1820 LM5032 Interleaved Boost Converter (Application Report No. SNVA335a). Retrieved October 10, 2020, from Texas Instruments website: https://www.ti.com/lit/an/snva335a/snva335a.pdf

Gunawan, T. (2009). Two-Phase Boost Converter. Master thesis. California Polytechnic State University.

Kumar, R., Singh, B., Chandra, A., \& Al-Haddad, K. (2015, September). Solar PV array fed water pumping using BLDC motor drive with boostbuck converter. In 2015 IEEE Energy Conversion Congress and Exposition (ECCE) (pp. 5741-5748). IEEE.

Kamil, M. (2010). Grid-connected solar microinverter reference design using a dsPIC $®$ digital signal controller. Microchip Application Notes AN1338.

Nguyễn Ngọc Trung. (2012). Tìm điểm công suất cực đại của pin mặt trời (Luận văn cao học). Trường Đại học Kỹ thuật Công nghệ Thành phố Hồ Chí Minh.
Nguyễn Văn Tấn, Dương Minh Quân, Trần Anh Tuấn, Phạm Văn Kiên, Lê Hồng Lâm và Hà Hải Long. (2018). So sánh các thuật toán bắt điểm công suất cực đại bằng phương pháp mô phỏng và thực nghiệm. Tạp chí Khoa họ và Công nghệ Đại học Đà Nẵng, Quyển 2 (11), 64-68.

Oprea, S., Rosu-Hamzescu, M., \& Radoi, C. (2014, October). Implementation of simple MPPT algorithms using low-cost 8-bit microcontrollers. In Proceedings of the 2014 6th International Conference on Electronics, Computers and Artificial Intelligence (ECAI) (pp. 31-34). IEEE.

Rao, P., Siraswar, V., \& Pimple, B. B. (2017, February). Efficient implementation of MPPT and comparison of converter for variable load Solar PV system. In 2017 Second International Conference on Electrical, Computer and Communication Technologies (ICECCT) (pp. 16). IEEE.

Rosu-Hamzescu, M., \& Oprea, S. (2013). Practical guide to implementing solar panel MPPT algorithms. Microchip Technology Inc, Application Note, AN1521.

De Stasi, F. (2015). Working with Boost Converters. Texas Instrument.

Vamja, R. V., \& Mulla, M. A. (2018, December). Solar pv fed induction motor driven water pumping system utilizing quadratic boost converter. In 2018 8th IEEE India International Conference on Power Electronics (IICPE) (pp. 1-6). IEEE.

Zdanowski, M., Rabkowski, J., \& Barlik, R. (2017). Highly-Efficient and Compact $6 \mathrm{~kW} / 4 \times 125 \mathrm{kHz}$ Interleaved DC-DC Boost Converter with $\mathrm{SiC}$ Devices and Low-Capacitive Inductors. Energies, 10(3), 363. 